\title{
Finding a Non-continuous Tube by Fuzzy Inference for Segmenting the MR Cholangiography Image
}

\author{
Chihiro Yasuba ${ }^{1}$, Syoji Kobashi ${ }^{1}$, Katsuya Kondo ${ }^{1}$, Yutaka Hata ${ }^{1,2}$, \\ Seturo Imawaki ${ }^{3}$, and Makoto Ishikawa ${ }^{3}$ \\ ${ }^{1}$ Graduate School of Engineering, Himeji Institute of Technology, \\ 2167, Shosha, Himeji, 671-2201, Japan \\ \{yasuba, kobashi, kondo, hata\}@comp.eng.himeji-tech.ac.jp \\ http://wwwj 3.comp.eng.himeji-tech.ac.jp/ \\ ${ }^{2}$ Chairman of BISC Special Interest Group in Medical Imaging, \\ The University of California at Berkeley, CA, USA \\ ${ }^{3}$ Ishikawa Hospital, 784, Bessho, Bessho-cho, Himeji, 671-0221, Japan
}

\begin{abstract}
In medical images, the tube-formed tissues such as the blood vessels, the trachea, and the pancreatic duct are sometimes partially masked because of the constriction, stones in the vessels, the pancreatic cancer, etc. Therefore, it is not easy to automatically segment the region of tubes (ROTs) from medical images for visualizing the structures by using conventional image segmentation methods, because inference of ROTs is difficult. In this paper, we propose a fuzzy rule-based augmented reality method for finding noncontinuous ROTs. We can obtain the ROT without extracting it. The physicians' procedure for finding the ROT can be eliminated by fuzzy inference techniques based on their knowledge. The employed knowledge is the intensity, the curve, and the radius of the ROTs. We apply the proposed method for finding the pancreatic duct from MR Cholangiography images. Through experimental results, we show that this method can successfully find the pancreatic duct from any data sets and it can clearly visualize the 3D shape of the ROT in MIP images.
\end{abstract}

\section{Introduction}

Tube-formed tissues such as the blood vessels, the trachea and the pancreatic duct are sometimes partially masked in medical images by the constriction, stones in vessels, the obliteration, etc. Therefore, it is not easy to automatically segment region of tubes (ROTs) from medical images for visualizing their structures by using conventional image segmentation methods. Generally, medical scanner such as X-ray computed tomography (X-ray CT) and magnetic resonance imaging (MRI) has been using for diagnosis. A volume data acquired from such scanner is composed of volumetric tomograms with detailed anatomical information. Today, ROTs are diagnosed using $3 \mathrm{D}$ visualization method; checking region of ROTs for every slice or maximum intensity projection (MIP) technique. However it is difficult to understand the shape 
and the location of ROTs by these methods. Therefore, it is necessary to extract ROTs from medical images. Many segmentation algorithms have been proposed for the ROT extraction from medical images. They are applied to the segmentation of the blood vessels from MR Angiography images or CT Angiography images [1], the trachea from CT images [2], and the bile duct from MR-C images [3]. Although these methods can segment the tube-formed tissue in some cases, they are not applicable in the noncontinuous ROTs case.

We propose the novel method for identifying the area of the non-continuous tube, and augmenting the area of ROTs. The proposed method is achieved by the fuzzy inference of illusory contours. The tube-formed tissue is expressed by using three fuzzy degrees, viz. the bend grade (curve), the intensity of tube-formed tissue, and the smoothness (radius). The medial line of tube can be traced by these fuzzy degrees. And it is robust because it can express ROTs without segmenting ROTs. In sec.2 we describe three fuzzy degrees. In sec.3, we apply this method to artificial images and MR-C images.

\section{Finding Non-continuous Tube}

This method traces the medial line of tubes based on illusory contour. Illusory contour is the non-continuous area [4]. For example, we consider that a line can be recognized by interpolating the non-continuous parts in the procedure as illustrated in Fig. 1. Step 1 finds the non-continuous part and, Step 2 extends the search region. In Step 3, we interpolate the non-continuous part to find another line that it may be connected with the previous line. The procedure for finding non-continuous tube is shown in Fig. 2.

This method is based on fuzzy inference technique [5]. It traces the medial nodes of tube as the medial line, from a starting node by using three fuzzy degrees, such as

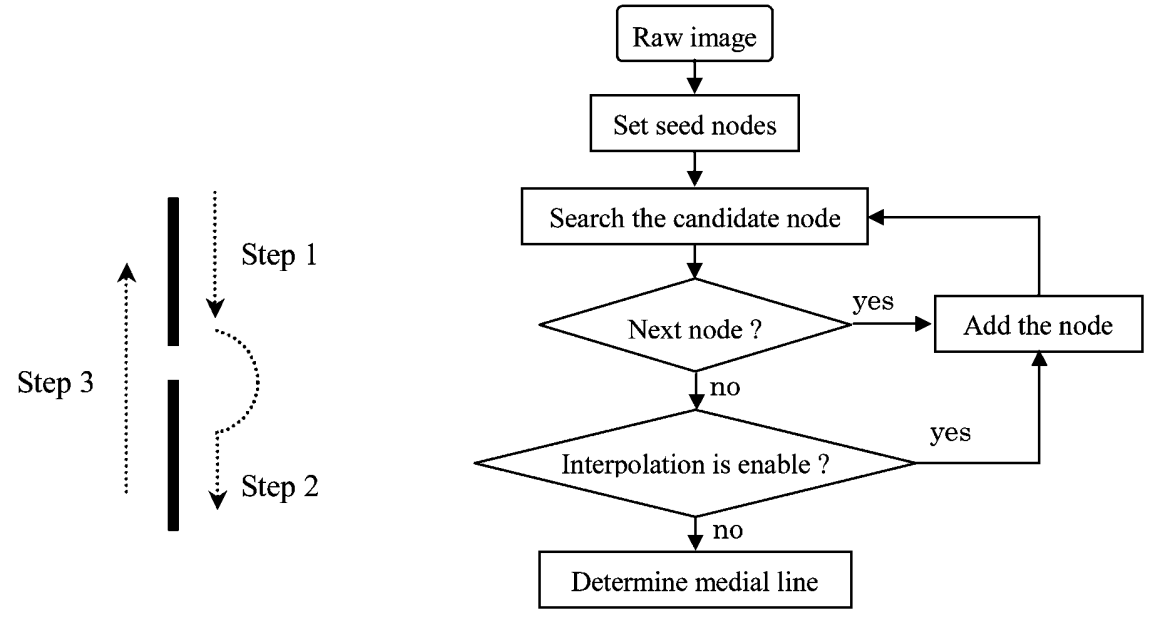

Fig. 1. Non- continuous tube

Fig. 2. Overall prosedure of our method 


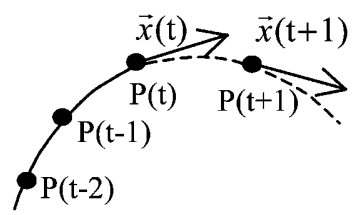

Fig. 3. Tangent vector at nodes

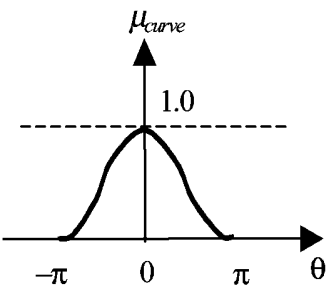

Fig. 4. Membership function for flexibility

curve, intensity and radius. When we cannot find the next node, the next node is searched in expanded search region. If we find the node that may be connected with the previous node, then we determine it as next node.

\subsection{The Degree of Curve}

The tube-formed tissue carves smoothly. The degree of the curve, which means the possibility that a target node is the next node, denotes the bend grade of a tube. We consider a line with two nodes, $P(t)$, and $P(t+1)$. The tangent vectors at $P(t)$ is $\vec{x}(t)$, as illustrated in Fig. 3. The orientational change of tangent vector can be formulated as

$$
\theta(\mathrm{t}+1)=\cos ^{-1}\left(\frac{\overrightarrow{\mathbf{y}}(\mathrm{t}) \cdot \overrightarrow{\mathbf{y}}(\mathrm{t}+1)}{|\overrightarrow{\mathbf{y}}(\mathrm{t})| \overrightarrow{\mathbf{y}}(\mathrm{t}+1) \mid}\right),
$$

where $\overrightarrow{\mathbf{y}}(\mathrm{t})$ denotes the differential of $\overrightarrow{\mathbf{x}}(\mathrm{t})$. Angle $\theta(\cdot)$ ranges between 0 and $\pi[\mathrm{rad}]$. Using the difference between $\overrightarrow{\mathbf{y}}(\mathrm{t})$ and $\overrightarrow{\mathbf{y}}(\mathrm{t}+1)$, we represent the knowledge of the curve with the fuzzy if-then rule;

IF $\quad \theta(\mathrm{t})$ is ' small',

THEN the degree $\mu_{\text {curve }}$ for direction to a next node is ' $h i g h$ '.

'small' is defined as the bell-shaped function in Fig. 4.

\subsection{The Degree of Radius}

The node of interest has similar radius of tube with the candidate node. The similarity is evaluated by means of the ratio of radius at the node of interest to the candidate node. Using the ratio, we represent the knowledge of the radius with the fuzzy if-then rule;

IF the ratio of radius of present node and radius of candidate node is ' $l$ ',

THEN the degree $\mu_{\text {radius }}$ for the radius of next node is ' $h i g h$ '. 


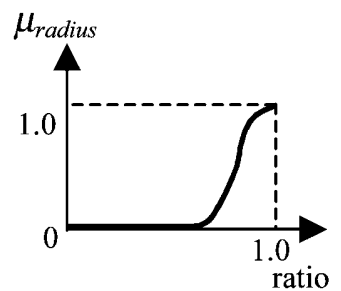

Fig. 5. Membership function for radius

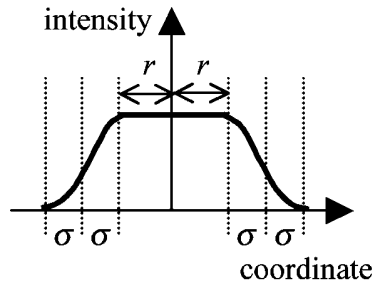

Fig. 6. Fitting function

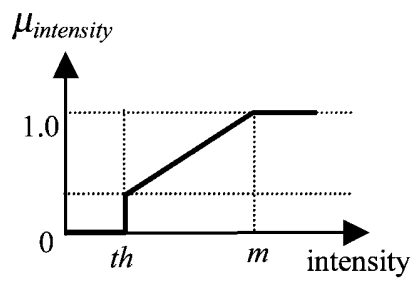

Fig. 7. Membership function of intensity

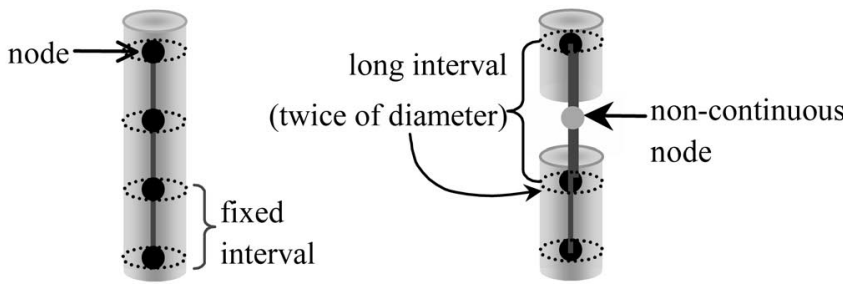

Fig. 8. Fixed and long interval

Fuzzy number ' 1 ' is defined as Zadeh's s-function in Fig. 5. The radius at each node is determined by fitting the fitting function using Zadeh's s-function shown in Fig. 6. $\sigma$ is the fixed value. The fitting function is made to fit ROTs by changing $r$. When the fitting function is most fitting, $r$ is determined as radius.

\subsection{The Degree of Intensity}

The center of tube-formed tissue has high intensity. Intensity of the end of the tubeformed tissue is lower than intensity of the half-long tube-formed tissue. Using intensity, we represent the knowledge of the intensity with the fuzzy if-then rule;

IF intensity is ' $h i g h$ ',

THEN the degree $\mu_{\text {intensity }}$ for next node is ' $h$ igh'.

The value ' $m$ ' is the mean of the intensity value of starting nodes, and ' $t h$ ' is the lower intensity threshold of tube- formed tissue. The membership function of intensity is represented in Fig. 7.

\subsection{Finding the Medial Line}

The medial line consists of nodes at a fixed interval. This line starts from two given nodes, and it is extended by evaluating three degrees, $\mu_{\text {curve }}, \mu_{\text {radius }}$, and $\mu_{\text {intensity. }}$. For each candidate points in a search region, a node with the highest fuzzy degree for next node is selected. The fuzzy degree for next node is defined as 


$$
\mu_{\text {next }}=\min \left\{\mu_{\text {curve }}, \mu_{\text {radius }}, \mu_{\text {int ensity }}\right\} \text {, }
$$

This process is iterated until $\mu_{\text {next }}$ is higher than 0.5 . If the degree is less than 0.5 , the nodes in search region that is twice diameter at node of interest are evaluated again to skip the non-continuous part as illustrated in Fig. 8. When the next node is found, the extension processing is carried out, otherwise tube finding process is finished.

\section{Experimental Results}

\subsection{Artificial images}

The proposed method was applied to two artificial images,

(1) 3D continuous bending tube (Fig. 9(a)),

(2) D non-continuous bending tube that the tube (a) is partly broken (Fig. 9(b)). The diameter of the tube is 11 voxels, and the length is 350 voxels. The interval of node was set 11 voxels. We set the parameters as follows; $t h=0, \sigma=1$. Two starting nodes were set to the lower end. The processing flow is shown in Fig. 9. The graph of the radius at each node is shown in Fig. 10. This shows that even if there is a noncontinuous portion, our method can trace the medial line of the tube.

\subsection{Pancreatic duct}

We applied our method to Magnetic Resonance Cholangiography (MR-C) images. $\mathrm{MR}-\mathrm{C}$ is an imaging technique, which non-invasively depicts the bile duct with high contrast [6]. MR-C has been used for diagnosing the shape and the direction of the bile duct and the pancreatic duct to reconstruct 3-D projection from MR-C volumetric image. Maximum intensity projection (MIP) technique is widely used [7]. This technique can provide interesting images, and help us to understand the shape and the location of the bile duct and the pancreatic duct, which is called ROI. However, the given images usually contain unnecessary tissues (e.g., fat or stomach) with high intensity. They prevent us to discriminate between the ROI and the unnecessary tissues. In order to understand the shape and location from MR-C volumetric images, it is necessary to find the ROI.

\subsubsection{Preliminaries of MR-C image}

We obtained MR-C images from Genesis Signa 1.5 Tesla MRI scanner (General Electric Medical Systems). The image acquisition method was single slice first spin echo with $\mathrm{TR}=75790.7 \mathrm{msec}$ and $\mathrm{TE}=188.2 \mathrm{msec}$. Field of view was $240 \mathrm{~mm}$. Matrix was $512 \times 512$. Thickness of the slice was $3.0 \mathrm{~mm}$. Voxel size was $0.47 \times 0.47 \times 3.0 \mathrm{~mm}^{3}$. Each of the volume data was composed of about 30 separated slices. The intensity value for all voxels of all ranged between 0 and 4095 . Figure 11 


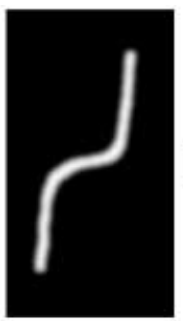

$1^{\text {st }}$

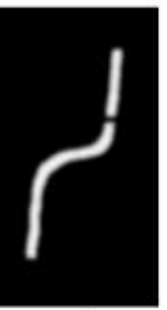

$1^{\text {st }}$

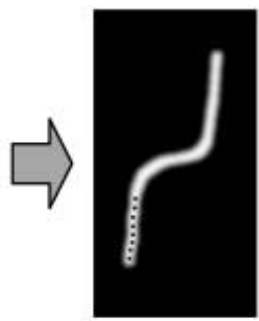

$6^{\text {th }}$

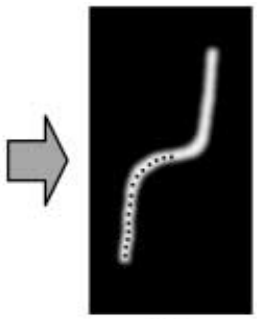

$13^{\text {th }}$

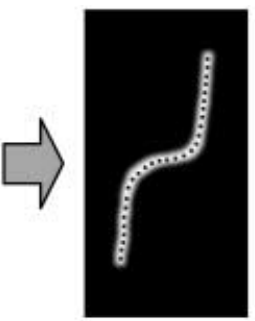

Final $\left(29^{\text {th }}\right)$

(a) the continuous tube data

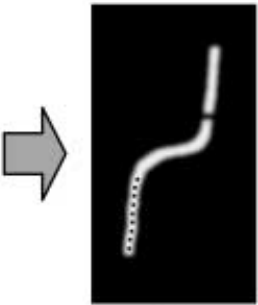

$7^{\text {th }}$

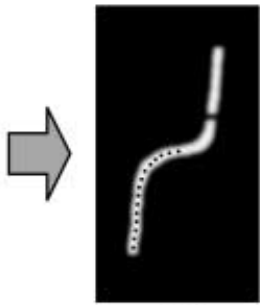

$13^{\text {th }}$

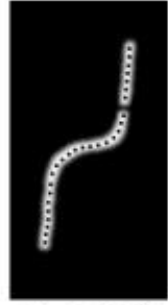

Final $\left(29^{\text {th }}\right)$

(b) the non-continuous tube data

Fig. 9. Experimental results (Artificial images)

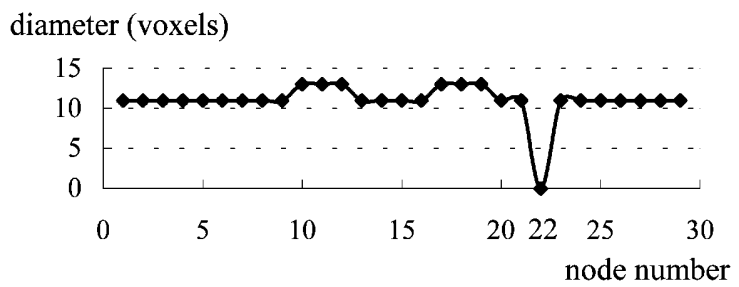

Fig. 10. The radius change on medial line (Fig. 9(b))

shows our coordinate system. Figure 12 shows raw MR-C slices. In raw images the pancreatic duct appeared as light gray.

\subsubsection{Finding the Pancreatic Duct}

Our method was applied to MR-C images of three patients for finding the pancreatic duct. The interval of node was set 6 voxels. Two starting nodes were set to the end of the node near the bile duct. We set the parameters as follows: $t h=100, \sigma=2$. We show the finded medial line of the pancreatic duct in Fig. 12. Black points in this figure indicate the nodes consisting of medial line. This method traced the medial line of the pancreatic duct. Figure 13 shows MIP images that the region of the pancreas duct was emphasized. This figure shows that the pancreatic duct can be easily observed. 


\section{Conclusions}

In this paper, we proposed the method for finding the non-continuous tube. This method expressed the findion process of the illusory contour by using three fuzzy degrees; curve, intensity and radius. These parameters represent the feature of tubeformed tissue. The experimental results showed that our method could find the noncontinuous tube-formed tissue as well as the continuous tube-formed tissue. The experimental results showed that this method could segmentation the pancreatic duct. The future work is to develop the method for finding tube with branch.

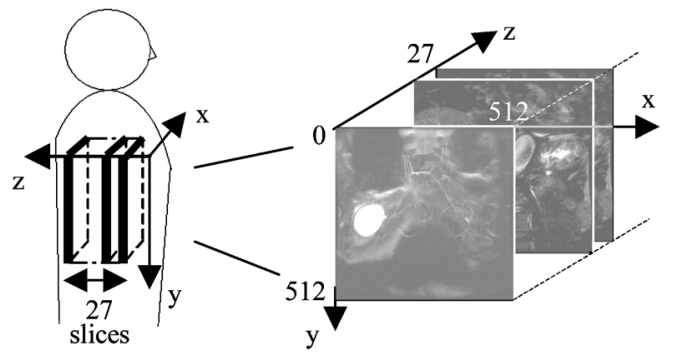

Fig. 11. Coordinate system

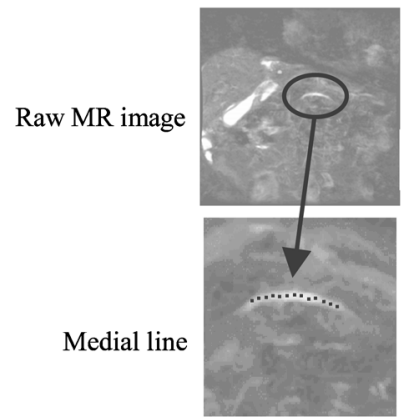

$\# 10 / 27$

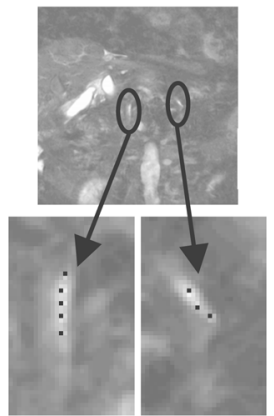

$\# 13 / 27$

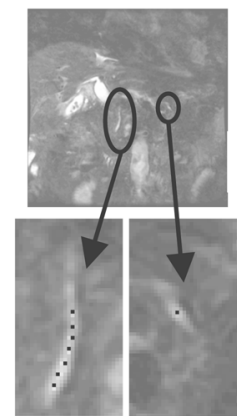

$\# 14 / 27$

Fig. 12. Raw MR-C images and finding the medial line of the pancreatic duct (The pancreatic duct is shown by the round mark.)

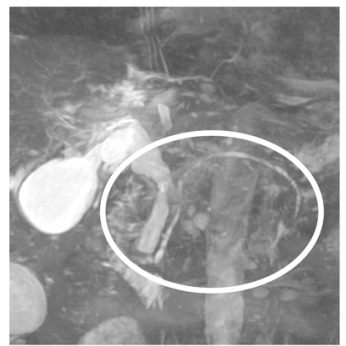

(a) Raw MIP image

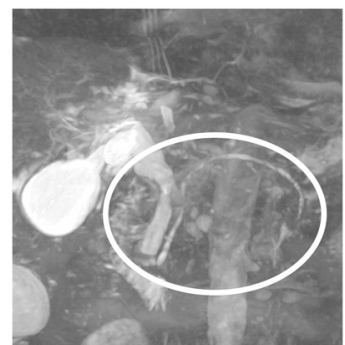

(b) Resultant MIP image

Fig. 13. MIP images for raw data and resultant data 


\section{Acknowledgement}

This research is supported in part by Ishikawa Hospital Grant and the BISC Program of UC Berkeley.

\section{References}

1. Kobashi, S., Kamiura, N., Hata, Y., Miyawaki, F,. Miyawaki: MR angiography Image Segmentation Aided by Fuzzy Information Granulaion, Proceding of the Sixth International Workshop on Parallel Image Processing and Analysis (1999) 9-14

2. Brown, M. S., Goldin, G. J., McNitt-Gray, M. F., Greaser, L. E., Sapra, A., Li, K., Sayre, J. W., Martin, K., Aberle, D. R.: Knowledge-based segmentation of thoracic computed tomography images for assessment of split lung function, Medical Physics, Vol. 27. 3 (2000)

3. Yasuba, C., Kobashi, S., Hata Y.: 3-D Visualization of the Cholecyst and the Bile duct Using Fuzzy Clustering, Knowledge-Based Intelligent Information Engineering Systems \& Allied Technologies, (2001) 446-450

4. Victor, J. D., Conte, M. M.: Illusion Contour Strength Does Not Depend on the Dynamics Or Relative Phase of the Inducets, Vision Research 40, (2000) 3475-3483

5. Zadeh, L. A.:Fuzzy Sets and Applications, Jhon Wiley and Sons. Inc., 1987.

6. Watanabe, Y., Dohke, M., Ishimori, T., Amoh, Y., Oda, K., Okumura, A., Mitsudo, K.,. Dodo, Y.:High-resolution MR Cholangiopancreatography, Critical Reviews in Diagnostic Imaging, Vol. 39. 2-3 (1998) 115-258

7. Lichtenbelt, B., Crane, R., Naqvi, S.: Introduction to Volume Rendering, Hewlette-Packard Co lette-Packard Company, (1998) 49, 50, 135, 136 